\title{
Nucleation in suspensions of anisotropic colloids
}

\author{
Tanja Schilling ${ }^{\mathrm{a}, *}$, Daan Frenkel $^{\mathrm{b}}$ \\ ${ }^{a}$ Institut für Physik, Johannes Gutenberg Universität Mainz, 55099 Mainz, Germany \\ $\mathrm{b}^{\mathrm{b}}$ FOM Institute for Atomic and Molecular Physics, Kruislaan 407, 1098 SJ Amsterdam, The Netherlands
}

Available online 7 April 2005

\begin{abstract}
We report Monte Carlo studies of liquid crystal nucleation in two types of anisotropic colloidal systems: hard rods and hard ellipsoids. In both cases we find that nucleation pathways differ strongly from the pathways in systems of spherical particles. Short hard rods show an effect of self-poisoning. This part of the article is based on a previous publication [T. Schilling, D. Frenkel, Self-poisoning of crystal nuclei in hard-rod liquids, Phys. Rev. Lett. 92 (2004) 085505]. When a crystallite forms, its surfaces are covered preferentially by rods which align perpendicular to the surface. Therefore subsequent growth is stunted. Hard, almost spherical ellipsoids can be compressed to very high densities without crystallization—in contrast to hard spheres, which crystallize easily. When forced to crystallize, ellipsoids form very loosely aggregated nuclei.

In both cases nucleation pathways are complex and it is therefore difficult to define an appropriate reaction coordinate. The common strategies of investigation of nucleation problems (i.e. definition of a coordinate and then sampling barrier crossing with well-known techniques) fail in these systems.
\end{abstract}

() 2005 Elsevier B.V. All rights reserved.

Keywords: Nucleation; Monte Carlo simulation; Anisotropic colloids

\section{Introduction}

In recent experimental work and simulations [2] it has been shown, that the disordered phase of nearspherical hard ellipsoids can reach densities similar to densest crystal phase. This stands in contrast to hard spheres, for which the disordered phase reaches a packing fraction $\phi \approx 0.64$ whereas the densest crystal structure has a packing fraction of $\phi \approx 0.74$. Hard spheres tend to crystallize very easily, when com-

\footnotetext{
* Corresponding author. E-mail address: schillit@uni-mainz.de (T. Schilling).
}

pressed beyond liquid-crystal coexistence. The results of Donev et al. indicate that this is different for ellipsoids.

Recently the pathways and rates of crystal nucleation have been obtained by computer simulation for several systems of spherical colloidal particles [3]. When spherical particles crystallize, the crystal nuclei that form tend to be roughly spherical $[3,4]$. In contrast, non-spherical molecules (as, for example, many proteins or the building blocks of liquid crystals) may form crystals that are strongly anisometric. This has two reasons: First of all, the solid-liquid interfacial free-energy density may be very different for crys- 
tal faces parallel and perpendicular to the molecular axes. As a consequence, the equilibrium shape of crystal nuclei is non-spherical. In addition, once crystals grow beyond the size of the critical nucleus, the rate at which molecules are incorporated into the crystal may depend strongly on the nature of the crystal face.

In this article we present results of simulations of crystal nucleation both in systems of ellipsoids and rods. The results on rods have been published previously [1].

\section{Model and simulation technique}

We studied two types of particles: hard rods and hard ellipsoids. The rods are modeled as hard spherocylinders with a diameter $D$ and a cylindrical segment of length $L$. In our simulations, we considered 2400 rods with an aspect ratio $L / D=2$. Short, hard spherocylinders have a relatively simple phase diagram [5]: at low pressures, the system forms an isotropic fluid, at high pressures it forms an orientationally ordered crystal. The transition between the two phases is of first order. At a pressure $p=5.64 k_{B} T / D^{3}$, the isotropic fluid coexists with the crystal phase.

The hard ellipsoids were ellipsoids of revolution with an aspect ratio $a / b=1.25, b=c=1$. We considered 1300 ellipsoids in most cases and some samples of 2700 ellipsoids. Ellipsoids of this aspect ratio exhibit three phases [6]: at low pressures they are positionally and orientationally disordered. At $p=$ $14.34 k_{B} T / 8 a b^{2}$ there is a transition to the positionally ordered, but orientationally disordered rotator phase. At $p \approx 60 k_{B} T / 8 a b^{2}$ orientational order sets in.

In the case of the spherocylinders, we were interested in the nucleation of a crystal from the metastable fluid phase. To study this phenomenon, we compressed the fluid beyond coexistence, to a pressure of $p=6 k_{B} T / D^{3}$. At this pressure, the chemical potential difference between the (metastable) fluid and the solid phases is approximately $0.2 k_{B} T$ per particle. In spite of the fact that, at this pressure, the solid is more stable than the liquid, spontaneous crystallization never occurred on the time-scales of our (quite long) simulations. However, at higher supersaturations (e.g., $p=8, \Delta \mu=1.3 k_{B} T /$ particle) spontaneous crystallization did occur on the timescale of a simulation. By working at $p=6$, a pressure where spontaneous crystallization is suppressed, we can study the free energy and the structure of small crystal nuclei using the biased sampling techniques described in Refs. [3,4].

The basic idea behind these techniques is the following: one defines a local order parameter, which distinguishes the liquid phase from all possible solid phases which might occur along the nucleation path. With this parameter clusters of "solid-like" particles are identified. Then the simulation is biased such that it produces on average a given size for the largest solidlike cluster in the system. For a series of biased simulations, histograms of cluster size and structure are measured and combined into the complete nucleation barrier.

In order to follow the formation of crystallites from solution, we chose the following order-parameter to distinguish particles in a crystalline environment from those in the liquid phase:

Two particles $i$ and $j$ are said to belong to the same crystallite if: (a) their surface-to-surface distance is less than $0.5 D$ and (b) the dot product of the unit vectors along their respective axes satisfies $\left|\mathbf{u}_{i} \cdot \mathbf{u}_{j}\right|>$ 0.995. (The choice of the exact value of this threshold is arbitrary, as long as it distinguishes between the solid and the fluid phase. We found our results to be stable against variation of this value.) As the criterion only considers the relative orientation of neighboring particles, it does not distinguish between crystalline, smectic and (dense) nematic structures. This means that we do not impose strong constraints on the structure of the crystal nucleus, but let the system find its own path.

We denote the average number of clusters consisting of $n$ particles by $N_{n}$. The probability to find a cluster of size $n$ per unit volume is equal to $(N / V)\left(N_{n} / N\right)$, where $N$ is the total number of particles in the system. We measure $P(n) \equiv N_{n} / N$, and define an intensive free energy barrier for the formation of a crystal nucleus of $n$ particles as $\Delta G(n)=$ $-k_{B} T \ln P(n)$. To probe $P(n)$ for $n>15$, we use biased, multi-histogram simulations (for details, see Ref. [3]).

In the case of the ellipsoids, we were interested in the nucleation of a rotator crystal from the isotropic phase. As a criterion to distinguish between the positionally disordered fluid and the positionally ordered rotator phase, we chose two types of bond-order para- 
meters: (a) We used $\vec{q}_{6} \cdot \vec{q}_{6}$ as it is defined, for example, in the work of ten Wolde [4]. (b) For each particle we counted the number of nearest neighbors pairs, which "lie opposite each other", i.e. the number of nearest neighbor bonds which form a straight line. A particle was counted as "solid-like" if five or more pairs of bonds formed an angle between $165^{\circ}$ and $195^{\circ}$. The latter parameter is computationally much cheaper than the former.

The ellipsoid system was compressed at $p=$ $20 k_{B} T / 8 a b^{2}$ and $p=25 k_{B} T / 8 a b^{2}$, which corresponds to $\Delta \mu=0.2 k_{B} T /$ particle and $\Delta \mu=0.5 k_{B} T /$ particle, respectively.

\section{Results}

\subsection{Spherocylinders}

We find that we can grow large, ordered clusters of up to 80 particles. However we find that most particles in the ordered cluster are located in a single hexagonally ordered layer. As we bias the cluster-size distribution towards larger crystallites, we observe no tendency to form crystallites that contain multiple layers. To be more precise: a few particles may order on top of the crystallite, but these embryonic new layers quickly dissolve again. All growth of the cluster concentrates on the edges, and the free energy of the cluster rises monotonically with size (see Fig. 1). In other words: the system never crosses a nucleation barrier beyond which crystallites would grow spontaneously.

Interestingly, a rough analysis of the data shown in Fig. 1 suggests that a flat crystallite is not the most stable one. (For details see [1].) We should expect multi-layer clusters to be more stable than flat disks. The fact that, in our simulations, we observe the formation of disk-like crystallites suggests that there are kinetic reasons that inhibit the formation of multi-layer crystallites.

Fig. 2 shows the angular distribution of particles in the liquid close to the top surface (the surface perpendicular to the director field) and the side surface (the surface parallel to it) of a perfect crystal of hard spherocylinders. Both distribution peak for $|\cos \theta|=1$, where particles are aligned with the director. However, for the top surface there is also a broad peak at $\cos \theta=0$, which indicates that a large fraction of the particles align parallel to the surface.

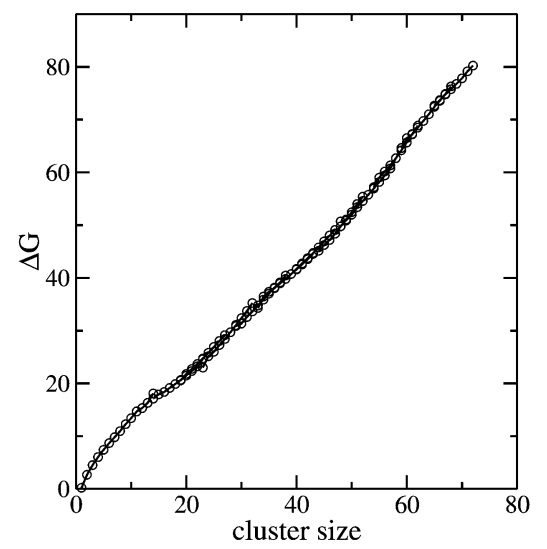

Fig. 1. Size dependence of the free energy of a crystal nucleus in the hard rod system. For the low supersaturations that we studied, $\Delta G$ for a single crystalline layer grows monotonically with cluster size. In order to nucleate a stable crystal, a multi-layer nucleus must first form.

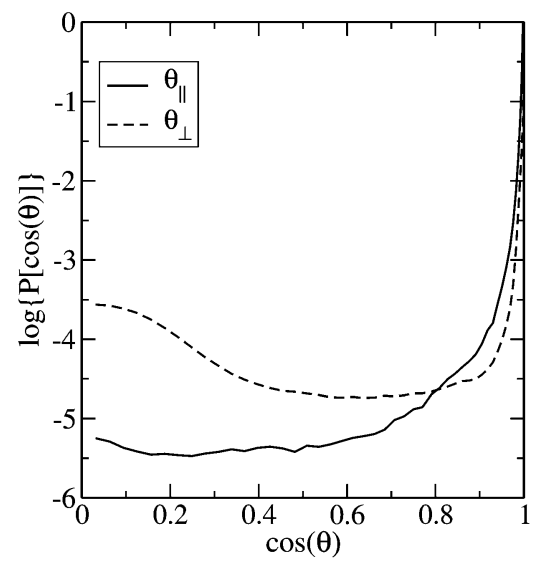

Fig. 2. Angular distributions of liquid particles close to a surface of a hard-rod crystal. $\theta_{\|}$denotes the surface parallel to the director field ("side surface"), $\theta_{\perp}$ the surface perpendicular to it ("top surface").

The preference of particles to lie flat on the crystal surface facilitates growth of the side surface, but it makes growth of the top/bottom surface difficult. Particles have to overcome a barrier of roughly $1.5 \mathrm{kT} /$ particle in order to stand up on the surface and align with the director. In contrast, incorporation of particles on the side face of a crystal is relatively easy. Indeed, we find no inhibition of the lateral growth of spherocylinder crystals, even when the growth of additional layers is effectively suppressed.

Recent work by Downton and Allen indicates that substrate roughness has a marked effect on the an- 
gular distribution functions of "near-surface" particles [7]. In our work, we have not been able to investigate the dependence of surface roughness on supersaturation, because the simulations were done in a nonequilibrium situation. However, it would be very interesting to investigate how supersaturation influences nucleation behavior via the roughness of the lamellae.

Recent experiments by Maeda and Maeda [8] on the isotropic-to-smectic transition in colloidal hard rods of $\beta$-FeOOH rods, showed that these particles tend to form disk-like crystallites. The present simulations suggest that this experimental observation is a logical consequence of the fact that, at sufficiently high supersaturation, multilayer growth is still kinetically inhibited, due to orientational "self-poisoning" of the top and bottom growth surfaces, whereas lateral growth proceeds relatively unhampered.

However, our results hold only for short rods. The formation of smectic and solid aggregates of longer rods $(L / D \geqslant 3)$ proceeds via the nematic phase and therefore follows a different pathway.

\subsection{Ellipsoids}

Before discussing nucleation, we would like to refer to a striking difference between compression of a disordered sample of ellipsoids and of spheres. Fig. 3 shows the equation of state of hard ellipsoids. The dotted branch was produced by expansion of an ordered crystal, the solid branch by compression of a liquid. In contrast to hard spheres, the disordered phase can be compressed to very high densities without spontaneous crystallization. Investigation of orientational order and bond order shows that the compressed phase is truly disordered.

We then performed a nucleation simulation similar to the one described before on the overcompressed disordered phase at $p=20 k_{B} T / 8 a b^{2}$ and $p=$ $25 k_{B} T / 8 a b^{2}$ with a bias on the size of the largest cluster. Clusters where identified by bond-order. A particle was counted as solid, if $\vec{q}_{6} \cdot \vec{q}_{6}>0.45$ for six or more bonds. Fig. 4 shows $\Delta G$ versus clusters size. In the case of low overcompression (X-symbols) we could not reach the critical nucleus size within several weeks of simulation time. For high overcompression (circles) the barrier reaches a plateau at a cluster size of roughly 20 particles. The clusters, however, are not compact (see Fig. 5).

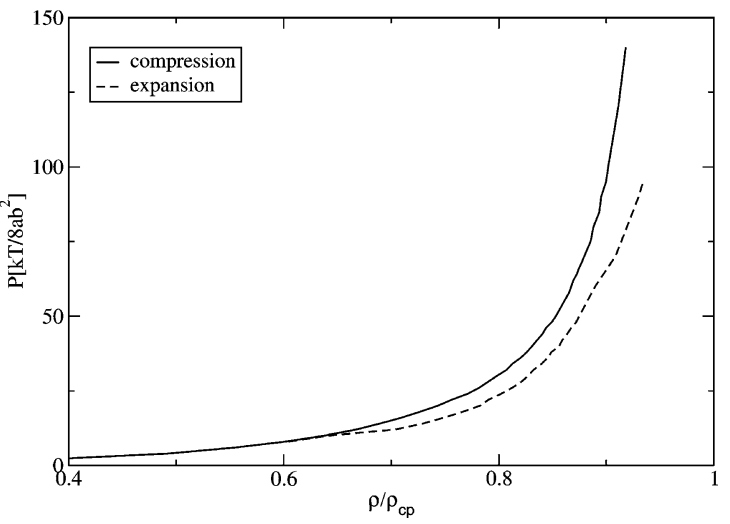

Fig. 3. Equation of state of hard ellipsoids. Dashed line: expansion from the crystalline phase. Solid line: compression from the liquid phase.

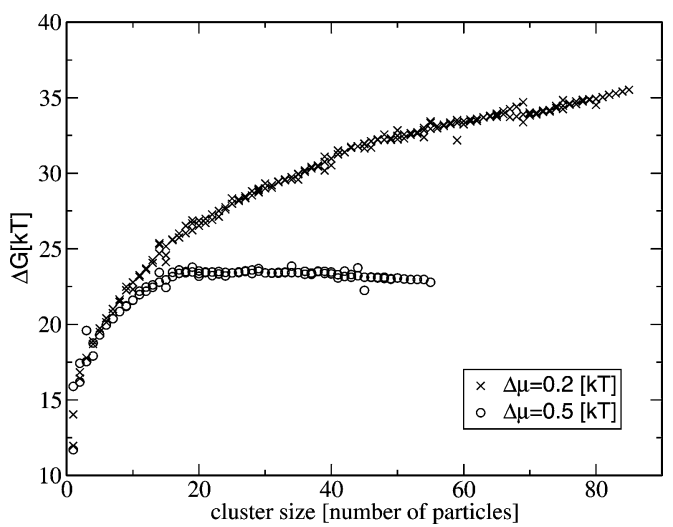

Fig. 4. Nucleation barriers of hard ellipsoids at $\Delta \mu=$ $0.2 k_{B} T /$ particle (crosses) and $\Delta \mu=0.5 k_{B} T /$ particle (circles).

In order to be able to collect significantly more statistics, we chose to repeat the simulations with the simpler order parameter described above. We managed to crystallize the system. However the intermediate structures were very large (almost spanning the entire system) and the slopes in the umbrella sampling windows did not match. This indicates that the choice of reaction coordinate is inappropriate.

\section{Conclusion}

We conclude that the pathways of crystal nucleation in systems of anisotropic particles are much more complex than in spherical systems. Therefore it is not possible to guess an appropriate reaction coor- 


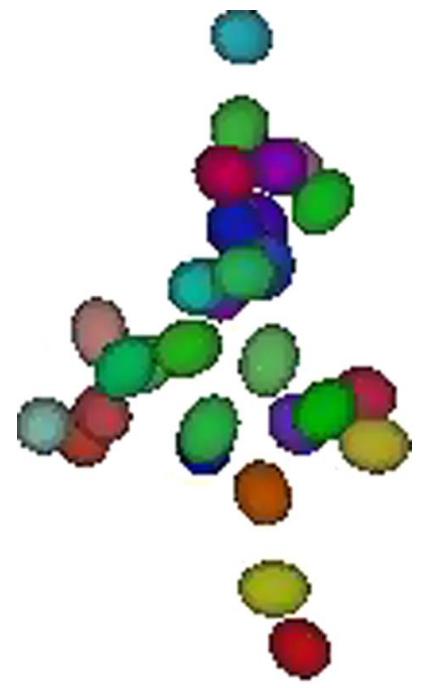

Fig. 5. Snapshot of a nucleus at $p=25 k_{B} T / 8 a b^{2}$.

dinate before hand and then apply standard techniques of simulating barrier crossing.

\section{Acknowledgements}

This work is part of the research program of "Stichting Fundamenteel Onderzoek der Materie
(FOM)" which is financially supported by "Nederlandse Organisatie voor Wetenschapelijke Onderzoek (NWO)".

\section{References}

[1] T. Schilling, D. Frenkel, Self-poisoning of crystal nuclei in hardrod liquids, Phys. Rev. Lett. 92 (2004) 085505.

[2] A. Donev, I. Cisse, D. Sachs, et al., Improving the density of jammed disordered packings using ellipsoids, Science 303 (2004) 990.

[3] S. Auer, D. Frenkel, Prediction of absolute crystal-nucleation rate in hard-sphere colloids, Nature 409 (2001) 1020.

[4] P.R. ten Wolde, M.J. Ruiz-Montero, D. Frenkel, Simulation of homogeneous crystal nucleation close to coexistence, Faraday Discuss. 104 (1996) 93.

[5] P. Bolhuis, D. Frenkel, Tracing the phase boundaries of hard spherocylinders, J. Chem. Phys. 106 (1997) 666.

[6] D. Frenkel, B.M. Mulder, The hard ellipsoids-of-revolution fluid. I. Monte Carlo simulations, Mol. Phys. 100 (1985/2002) 201.

[7] M. Downton, M. Allen, Computer simulation of liquid crystal surface modification, preprint available from http: //eprints.csc.warwick.ac.uk/.

[8] H. Maeda, Y. Maeda, Liquid crystal formation in suspensions of hard rodlike colloidal particles: direct observation of particle arrangement and self-ordering behavior, Phys. Rev. Lett. 90 (2003) 018303. 